\title{
Variational relation problems: existence of solutions and fixed points of contraction mappings
}

\section{Abdul Latif $f^{*}$ and Dinh The Luc ${ }^{2}$}

"Correspondence: alatif@kau.edu.sa ${ }^{1}$ Faculty of Science, King Abdulaziz University, P.O. Box 80203, Jeddah, 21589, Saudi Arabia

Full list of author information is available at the end of the article

\begin{abstract}
We propose a new approach to study variational relation problems. Namely, we apply Mizoguchi and Takahashi's fixed point theorem of contraction mappings and an error bound of a system of linear inequalities to establish existence conditions for a variational relation problem in which the variational relation linearly depends on the decision variable. Then we develop an algorithm to compute a solution of a linear variational relation problem.
\end{abstract}

MSC: 49J52; 47H10

Keywords: contraction mapping; fixed point; error bound; variational relation problem

\section{Introduction}

We consider the following variational relation problem: find $\bar{x} \in X$ such that

(1) $\bar{x}$ is a fixed point of $S$, that is, $\bar{x} \in S(\bar{x})$,

(2) $R(\bar{x}, y)$ holds for every $y \in T(\bar{x})$,

where $X$ and $Y$ are nonempty sets, $S$ is a set-valued mapping from $X$ to itself, $T$ is a setvalued mapping from $X$ to $Y$, and $R(x, y)$ is a relation linking $x \in X$ and $y \in Y$. In an abstract setting, the relation $R$ is represented by a subset of the product space $X \times Y$ so that $R(x, y)$ holds if and only if the point $(x, y)$ belongs to that set. In practice, however, $R$ is often given by equality/inequality of real-valued functions, or by inclusion/intersection of set-valued mappings on $X \times Y$. For instance, given a real-valued function $\phi$ on $X \times Y$, a variational relation can be defined by any of the following equality and inequalities: $\phi(x, y)=0, \phi(x, y) \neq 0, \phi(x, y)>0$ or $\phi(x, y) \geq 0$. When two set-valued mappings $G_{1}$ and $G_{2}$ are given on $X \times Y$ with values in a nonempty set $Z$, a variational relation can be defined by any of the following inclusions and intersections: $G_{1}(x, y) \subseteq G_{2}(x, y), G_{1}(x, y) \nsubseteq G_{2}(x, y)$, $G_{1}(x, y) \cap G_{2}(x, y)=\emptyset$ or $G_{1}(x, y) \cap G_{2}(x, y) \neq \emptyset$. A mixture of the above relations is also possible.

The variational relation problem was introduced in [1] and studied in a number of recent works [2-13]. It encompasses a large class of problems of applied mathematics including optimization problems, variational inequalities, variational inclusions, equilibrium problems etc., and offers a unifying treatment of problems that come from different areas and have a similar structure. Existence conditions of solutions to variational relation problems were analyzed in great generality, the stability of solutions of a parametric variational re-

(อ2013 Latif and Luc; licensee Springer. This is an Open Access article distributed under the terms of the Creative Commons Attribution License (http://creativecommons.org/licenses/by/2.0), which permits unrestricted use, distribution, and reproduction in any medium, provided the original work is properly cited. 
lation was also studied with respect to the continuity of set-valued mappings, and very recently a numerical method was developed to solve variational relation problems when the data are linear [14]. As far as we know, all conditions established for existence of solutions of variational relation problems in the above cited papers utilize intersection theorems or fixed point theorems involving the KKM property of set-valued mappings in one or another form [15]. In the present paper, we wish to give existence conditions by exploiting Mizoguchi and Takahashi's fixed point theorem for contraction mappings and propose an algorithm to compute solutions to (VRP), which seems to be new in the theory of variational relations. Actually, we shall study a particular model of (VRP) in which $R$ linearly depends on the decision variable $x$. Throughout we assume

- $X$ and $Y$ are nonempty closed sets in the finite dimensional Euclidean spaces $\mathbb{R}^{n}$ and $\mathbb{R}^{m}$, respectively,

- $S(x)=X$ for every $x \in X$,

- $R(x, y)$ holds if and only if $A x-g(y) \leq 0$ with $A$ a $k \times n$ matrix, $g$ a vector function from $Y$ to $\mathbb{R}^{k}$.

Thus, our problem consists of finding a vector $\bar{x} \in X$ such that $A \bar{x} \leq g(y)$ for all $y \in T(\bar{x})$. This problem can also be transformed to a quasi-variational inequality problem or a quasiequilibrium problem [16-20], but its resolution, as far as we know, is not known, because a general monotonicity hypothesis often requested for quasi-variational inequality problems and quasi-equilibrium problems is not satisfied. If we define a set-valued mapping $\Gamma: X \rightrightarrows X$ by

$$
\Gamma(x)=\{z \in X: A z \leq g(y) \text { for all } y \in T(x)\},
$$

then the (VRP) above is equivalent to the fixed point problem: find $\bar{x} \in X$ such that $\bar{x} \in$ $\Gamma(\bar{x})$. Essentially we shall exploit this equivalent formulation of (VRP) to establish existence conditions and to develop a solving method.

The paper is structured as follows. In the next section, we present two preliminary results which constitute major tools of our study: a fixed point theorem by Mizoguchi and Takahashi [21], which generalizes the Nadler fixed point principle [22], and an error bound or Hoffman's constant for a system of linear inequalities (see [17, 19, 23-28]). Section 3 is devoted to sufficient conditions for existence of solutions of (VRP). In the last section, we propose an algorithm to compute a solution of (VRP) and illustrate it by some numerical examples.

\section{Preliminaries}

\section{Contraction mappings}

The famous Banach contraction principle states that if $(X, d)$ is a complete metric space and if $f$ is a real contraction function on $X$, then $f$ has a fixed point. This principle was generalized to the case of set-valued mappings by Nadler [22] and Markin [29] with the help of the Hausdorff distance. Since then a lot of investigation has been made in order to weaken the contraction hypothesis (see [21,30-34] and many references given in these). In the present paper, we are particularly interested in a theorem by Mizoguchi and Takahashi [21], which can elegantly be applied to our model. Let us recall it in details and make a discussion on its generalization. 
Let $D_{1}$ and $D_{2}$ be nonempty subsets of $X$. The Hausdorff distance between $D_{1}$ and $D_{2}$ is defined by

$$
h\left(D_{1}, D_{2}\right)=\max \left\{\sup _{x \in D_{1}} d\left(x, D_{2}\right) ; \sup _{y \in D_{2}} d\left(y, D_{1}\right)\right\}
$$

where $d\left(x, D_{i}\right)$ is the distance from $x$ to $D_{i}, i=1,2$. In $\mathbb{R}^{n}$ the Hausdorff distance can be given by

$$
h\left(D_{1}, D_{2}\right)=\inf \left\{r>0: D_{1} \subseteq D_{2}+B(0, r), D_{2} \subseteq D_{1}+B(0, r)\right\},
$$

where $B(0, r)$ is the closed ball of radius $r$ and centered at the origin of the space. Let $F$ be a set-valued mapping from $X$ to the space of closed subsets of $X$ and $\ell>0$. We say that $F$ is $\ell$-Lipschitz on $X$ if for every $x, x^{\prime} \in X$, one has

$$
h\left(F(x), F\left(x^{\prime}\right)\right) \leq \ell\left\|x-x^{\prime}\right\| .
$$

When $\ell<1$, an $\ell$-Lipschitz mapping is called a contraction mapping. We recall the following result by Mizoguchi and Takahachi [21], which generalizes Nadler's theorem on fixed points of a contraction mapping [22]: The set-valued mapping $F$, whose values are assumed nonempty closed and bounded, has a fixed point in $X$ if the following conditions hold:

(i) there exists a function $\gamma:(0, \infty) \rightarrow[0,1)$ such that $\lim \sup _{r \rightarrow t^{+}} \gamma(r)<1$ for each

$$
t \in[0, \infty) \text {; }
$$

(ii) for every $x, y \in X$, one has $h(F(x), F(y)) \leq \gamma(d(x, y)) d(x, y)$.

Further developments of this result can be found in [30, 35-38]. In particular the following theorem by Ciric (Theorem 2.2 [30]) is quite general: if $F$ has closed values and if there exists a real function $\phi:[0, \infty) \rightarrow[a, 1)$ for some $a \in(0,1)$ such that

- $\lim \sup _{s \rightarrow t^{+}} \phi(s)<1$ for every $t \geq 0$;

- for every $x \in X$, there is $y \in F(x)$ satisfying

$$
\begin{aligned}
& \sqrt{\phi(d(x, y))} d(x, y) \leq d(x, F(x)), \\
& d(y, F(y)) \leq \phi(d(x, y)) d(x, y),
\end{aligned}
$$

then $F$ admits a fixed point.

It is unfortunate that this theorem does not fully generalize Mizoguchi and Takahashi's theorem because in the latter theorem it is not requested that the function $\phi$ takes its values bigger than a strictly positive number $a$. Another observation we can make is the fact that Mizoguchi and Takahashi's theorem remains valid with the same argument even when $F$ has closed values that are not necessarily bounded.

\section{Error bound for a linear system}

We consider a linear system $A x \leq b$ in $\mathbb{R}^{n}$, where $A$ is a $k \times n$ matrix and $b$ is a $k$ vector. The solution set to this system is denoted $P$, which is a polyhedral convex set. It is clear that $x$ is a solution to this system if and only if $d\left(A x-b,-\mathbb{R}_{+}^{k}\right)=0$, where $\mathbb{R}_{+}^{k}$ is the positive octant of the space. When $x$ is not a solution, it is important to measure the distance 
from $x$ to the solution set $P$. This question has completely been studied starting with the famous Hoffman constant in [26] and subsequential works [23, 24, 27, 28] among many others. Here is a formula for the distance from $x$ to $P$ that can be obtained by elementary arguments from linear algebra (see [24]):

$$
d(x, P)=\max _{I \in \mathcal{I}} \max _{\lambda \in \mathbb{R}^{\mid I I} \backslash\{0\}} \frac{\left\langle\lambda, A_{I} x-b_{I}\right\rangle}{\left\|A_{I}^{T} \lambda\right\|},
$$

where $|I|$ denotes the cardinal of $I, \mathcal{I}$ the set of index subsets $I \subseteq\{1, \ldots, k\}$ satisfying two conditions below:

(1) $I$ is nonempty and the family of rows $a^{i}, i \in I$ of the matrix $A$ is linearly independent,

(2) there is some $y \in P$ such that $I$ is contained in the active index set $I(y)$ at $y$, that is, $a^{i} y=b_{i}, i \in I ; A_{I}$ is the submatrix of $A$ consisting of the rows $a^{i}, i \in I$, and $A_{I}^{T}$ is its transpose.

It follows that

$$
d(x, P) \leq \alpha d\left(A x-b,-\mathbb{R}_{+}^{k}\right)=\alpha\left\|(A x-b)^{+}\right\|,
$$

where $(A x-b)^{+}$is the vector obtained from $A x-b$ by substituting all negative components by zeros. The constant $\alpha>0$ is called an error bound of the system or Hoffman's constant. The best error bound, denoted $\alpha$ too, is obtained by maximizing the function $d(x, P) /\left\|(A x-b)^{+}\right\|$over $x \notin P$. It is given by the formula

$$
\alpha=\max _{I \in \mathcal{I}}[\gamma(I)]^{-1 / 2},
$$

where $\gamma(I)$ is the smallest Pareto eigenvalue of the matrix $A_{I} A_{I}^{T}$ (see [39]), or equivalently, it is the optimal value of the problem of minimizing $y^{T} A_{I} A_{I}^{T} y$ over the set $\left\{y \in \mathbb{R}_{+}^{|I|}:\|y\|^{2}=\right.$ $1\}$. If we denote by $P^{\prime}$ the solution set to the perturbed system $A x \leq b^{\prime}$, then

$$
h\left(P, P^{\prime}\right) \leq \alpha\left\|b-b^{\prime}\right\|
$$

whenever both $P$ and $P^{\prime}$ are nonempty.

\section{Existence conditions}

In this section we establish some sufficient conditions for existence of solutions to the variational problem (VRP) introduced in the beginning of Section 2. Mizoguchi and Takahashi's fixed point theorem and the error bound (2.1) are the main tools we use for this purpose. Given a real function $u(x)$ on $X$, we define a value function (called also a marginal function) of $u$ by $\beta(x)=\inf _{z \in T(x)} u(z)$. Stability and sensitivity of this function is one of the indispensable parts of the theory of optimization. We refer the interested reader to the books $[17,19,25]$ for greater details. The next result will be needed in the sequel.

Lemma 3.1 Assume that for every $x \in X$, the value function $\beta(x)$ is finite and that there are functions $\phi$ and $\psi$ from $[0, \infty)$ to $[0, \infty)$ with $\psi$ nondecreasing such that

$$
\begin{aligned}
& h(T(x), T(y) \leq \phi(d(x, y)) d(x, y) \\
& |u(x)-u(y)| \leq \psi(d(x, y)) d(x, y)
\end{aligned}
$$


for every $x, y \in X$. Then

$$
|\beta(x)-\beta(y)| \leq \phi(d(x, y)) \psi(d(x, y) \phi(d(x, y))) d(x, y) .
$$

Proof Let $x, y \in X$ be given. For each $\epsilon>0$, choose $z \in T(x)$ such that $\beta(x)>u(z)-\epsilon$. Choose also $w \in T(y)$ such that

$$
d(z, w) \leq h(T(x), T(y)) .
$$

Then by (3.2) we have

$$
\beta(y)-\beta(x) \leq u(w)-u(z)+\epsilon \leq \psi(d(w, z)) d(w, z)+\epsilon .
$$

Combining this, (3.1) and (3.3), we deduce

$$
\begin{aligned}
\beta(y)-\beta(x) & \leq \psi(h(T(x), T(y))) h(T(x), T(y))+\epsilon \\
& \leq \psi(d(x, y) \phi(d(x, y))) \phi(d(x, y)) d(x, y)+\epsilon .
\end{aligned}
$$

Switching the roles of $x$ and $y$ in the above inequality and taking into account the fact that $\epsilon$ is arbitrarily chosen, we obtain $|\beta(x)-\beta(y)| \leq \phi(d(x, y)) \psi(d(x, y) \phi(d(x, y))) d(x, y)$ as requested.

In the remaining part of this section, we assume that for every $x \in X$, the values $b_{i}(x)=$ $\inf _{z \in T(x)} g_{i}(z), i=1, \ldots, k$, are finite and that the system $A z \leq b(x), z \in X$ is consistent. The vector whose components are $b_{i}(x), i=1, \ldots, k$, is denoted $b(x)$.

Theorem 3.1 Assume that there are functions $\phi$ and $\psi_{i}, i=1, \ldots, k$, on $[0, \infty)$ to $[0, \infty)$ with $\psi_{i}$ nondecreasing and satisfying the following properties:

(i) $h(T(x), T(y)) \leq \phi(d(x, y)) d(x, y)$ for $x, y \in X$;

(ii) $\left|g_{i}(x)-g_{i}(y)\right| \leq \psi_{i}(d(x, y)) d(x, y)$ for $x, y \in X$ and $i=1, \ldots, k$;

(iii) $\limsup _{s \rightarrow t^{+}} \phi(s) \sqrt{\sum_{i=1}^{k} \psi_{i}(s \phi(s))^{2}}<\frac{1}{\alpha}$ for all $t>0$.

Then (VRP) admits a solution.

Proof The aim is to prove that the set-valued mapping $\Gamma$ has a fixed point. It is clear that $z \in \Gamma(x)$ if and only if $a^{i} z \leq g_{i}(y)$ for every $y \in T(x)$, or equivalently, $a^{i} z \leq b_{i}(x), i=1, \ldots, k$. By this, $\Gamma(x)$ consists of the solutions to the system $A z \leq b(x)$. According to (2.1), we have

$$
h(\Gamma(x), \Gamma(y)) \leq \alpha\|b(x)-b(y)\| \quad \text { for all } x, y \in X .
$$

We apply Lemma 3.1 to obtain

$$
\begin{aligned}
h(\Gamma(x), \Gamma(y)) & \leq \alpha \sqrt{\sum_{i=1}^{k}\left|b_{i}(x)-b_{i}(y)\right|^{2}} \\
& \leq \alpha \phi(d(x, y)) \sqrt{\sum_{i=1}^{k} \psi_{i}(d(x, y) \phi(d(x, y)))^{2}} d(x, y) .
\end{aligned}
$$


Consider the real function $\gamma(t)=\alpha \phi(t) \sqrt{\sum_{i=1}^{k} \psi_{i}(t \phi(t))^{2}}$ for every $t \geq 0$. Then the hypotheses of Mizoguchi and Takahashi's theorem are satisfied for the set-valued mapping $\Gamma$, by which it admits a fixed point. Consequently, (VRP) has a solution.

When the mapping $T$ and the function $g$ are Lipschitz, we derive the following result.

Corollary 3.1 Assume that $T$ is $\kappa$-Lipschitz and $g_{1}, \ldots, g_{k}$ is $\ell$-Lipschitz with $\kappa \ell<\frac{1}{\alpha \sqrt{k}}$. Then (VRP) has a solution.

Proof Set $\phi(t)=\kappa$ and $\psi_{i}(t)=\ell$ for $i=1, \ldots, k$ and $t \in[0, \infty)$ and apply Theorem 3.1.

Let us now consider the case when the function $g$ is affine, that is, $g(y)=C y+c$, where $C$ is a $k \times m$ matrix, $c$ is a $k$ vector, and the graph of $T$ is a convex polyhedral set, that is, $y \in T(x)$ if and only if $x$ and $y$ solve a linear system $P y \leq Q x+q$, where $P$ is a $k \times m$ matrix, $Q$ is a $k \times n$ matrix and $q$ is a $k$ vector. (VRP) with such linear data is called a linear variational relation problem. It was studied in [14] in which a numerical algorithm based on Delauney's triangulations is proposed for solving it. It is known that a linear variational problem may have no solutions (see Example 4.1). We wish to apply Theorem 3.1 to derive an existence condition for this model. The rows of the matrix $C$ are denoted $C^{1}, \ldots, C^{k}$, while the components of the vector $c$ are denoted $c_{1}, \ldots, c_{k}$. The best error bound for the system $P z \leq Q x+q$ is denoted $\alpha^{\prime}$.

Corollary 3.2 Assume that the error bounds $\alpha$ and $\alpha^{\prime}$, the matrices $Q$ and $C$ of the linear (VRP) satisfy

$$
\alpha \alpha^{\prime}\|Q\| \sqrt{\left\|C^{1}\right\|^{2}+\cdots+\left\|C^{k}\right\|^{2}}<1 .
$$

Then (VRP) has a solution.

Proof For every $x, y \in X$, by applying (2.1), we have

$$
\begin{aligned}
& \left|g_{i}(x)-g_{i}(y)\right|=\left|C^{i} x+c_{i}-\left(C^{i} y+c_{i}\right)\right| \leq\left\|C^{i}\right\| d(x, y), \\
& h(T(x), T(y)) \leq \alpha^{\prime}\|Q x+q-(Q y+q)\| \leq \alpha^{\prime}\|Q\| d(x, y) .
\end{aligned}
$$

Then, in view of Lemma 3.1, one deduces

$$
\left|b_{i}(x)-b_{i}(y)\right| \leq \alpha^{\prime}\|Q\|\left\|C^{i}\right\| d(x, y) \quad \text { for } i=1, \ldots, k
$$

It remains to apply Theorem 3.1 to conclude that (VRP) has a solution.

\section{An algorithm}

In this section, we consider a linear (VRP) as mentioned in the preceding section, namely we wish to find $\bar{x} \in \mathbb{R}^{n}$ such that $A \bar{x} \leq C y+c$ for every $y$ solution to the system $P y \leq Q \bar{x}+q$. As we have already noticed, this problem may have no solutions. Here is an example. 
Example 4.1 We consider a linear variational relation problem with $x \in[0,2]$ and $y \in \mathbb{R}$. The relation $R$ is defined by the system

$$
\left(\begin{array}{c}
-1 \\
-1 \\
1
\end{array}\right) x \leq\left(\begin{array}{c}
-1 \\
0 \\
0
\end{array}\right) y+\left(\begin{array}{c}
-1 \\
0 \\
2
\end{array}\right)
$$

and $T$ is defined by the system

$$
\left(\begin{array}{l}
0 \\
0 \\
1
\end{array}\right) x \leq\left(\begin{array}{c}
-1 \\
-1 \\
0
\end{array}\right) y+\left(\begin{array}{l}
4 \\
0 \\
1
\end{array}\right) .
$$

For every $x \in[0,2], y \in T(x)$ if and only if $0 \leq y \leq 4$. With $y=4$, it is clear that $R(x, y)$ does not hold, which means that (VRP) has no solutions.

Let us now describe an algorithm to solve (VRP).

Step 0 . Choose any $x_{0} \in X$, a small tolerance level $\epsilon>0$. Set $r=0$.

Step 1. For $i=1, \ldots, k$, solve

$$
\begin{array}{ll}
\text { minimize } & C^{i} y+c_{i} \\
\text { subject to } & P y \leq Q x_{r}+q, \\
& y \in Y .
\end{array}
$$

Let $b_{r}$ be the vector whose components are optimal values of the above programs.

Step 2. Solve

$$
\begin{array}{ll}
\text { minimize } & \left\|x_{r}-z\right\| \\
\text { subject to } & A z \leq b_{r}, \\
& x \in X .
\end{array}
$$

Let $z$ be an optimal solution of this program.

Step 3. Check $\left\|x_{r}-z\right\| \leq \epsilon$. If yes, stop. The optimal solution $z$ is considered as a solution of (VRP). Otherwise, set $r=r+1, x_{r}=z$ and return to Step 1 .

We have the following convergence property of the algorithm.

Proposition 4.1 Assume that the hypothesis of Corollary 3.2 holds. Then, for any $\epsilon>0$, the algorithm terminates after a finite number of iterations. If at some iteration the optimal value of the program in Step 2 is zero, then $x_{r}$ is a solution of (VRP). If not, with $\epsilon$ tending to 0 , the sequence of $x_{r}$ obtained in Step 3 converges to a solution of $(V R P)$.

Proof It is clear that $x_{r+1} \in \Gamma\left(x_{r}\right)$. If, for some $r$, the optimal value of the program in Step 2 is zero, then $x_{r} \in \Gamma\left(x_{r}\right)$ which means that $x_{r}$ is a fixed point of $\Gamma$, and hence a solution of (VRP). Moreover, since under the hypothesis of Corollary 3.2 the mapping $\Gamma$ is a contraction, the sequence $\left\{x_{r}\right\}_{r=0}^{\infty}$ converges to a fixed point of $\Gamma$, which is also a solution of 
(VRP). By this the algorithm terminates after a finite number of iterations when $\epsilon$ is strictly positive.

Below we give two examples to show how to perform the algorithm. In the first example, the algorithm terminates after two iterations and produces an exact solution. In the second example, we can obtain an approximate solution with any given strictly positive tolerance level.

Example 4.2 Consider a linear (VRP) with the following data: $X=[0,2], Y=\mathbb{R}^{2}$ and

$$
\begin{array}{ll}
A=\left(\begin{array}{c}
-1 \\
-1 \\
1
\end{array}\right), \quad C=\left(\begin{array}{cc}
-1 / 4 & 1 / 4 \\
0 & 0 \\
0 & 0
\end{array}\right), \quad c=\left(\begin{array}{c}
-1 \\
0 \\
2
\end{array}\right), \\
P=\left(\begin{array}{cc}
1 / 2 & 1 / 2 \\
1 / 2 & -1 / 2 \\
-1 & 0 \\
0 & -1
\end{array}\right), \quad Q=\left(\begin{array}{c}
1 \\
-1 \\
0 \\
0
\end{array}\right), \quad q=\left(\begin{array}{c}
0 \\
1 / 2 \\
0 \\
0
\end{array}\right) .
\end{array}
$$

Iteration 1 . We start the algorithm with $x_{0}=0$. The feasible set of the programs in Step 1 is given by the system $P y \leq q$, or equivalently,

$$
\begin{aligned}
& y_{1}+y_{2} \leq 0, \\
& y_{1}-y_{2} \leq 1, \\
& y_{1}, y_{2} \geq 0 .
\end{aligned}
$$

The three functions to minimize are respectively $\left(-y_{1}+y_{2}\right) / 4-1,0$ and 2, which implies that $b_{0}=(-1,0,2)^{T}$. In the next step, we minimize $|z|$ over the set $A z \leq b_{0}, z \in X$. The optimal solution is $z=1$. As $x_{0} \neq z$, it is not a solution of (VRP).

Iteration 2. We set $x_{1}=z=1$ and return to Step 1 to solve the three above mentioned functions over the set $P y \leq Q x_{1}+q$. The same optimal solutions are obtained and $b_{1}=$ $(-1,0,2)^{T}$. In Step 2, we minimize $|1-z|$ over the set $A z \leq b_{1}, z \in X$ and obtain the optimal solution $z=1$. Since $x_{1}=z$, the algorithm terminates and $x_{1}=1$ is a solution of (VRP). It is easy to see that $\Gamma$ is a (1/2)-contraction mapping, that is, $h\left(\Gamma(x), \Gamma\left(x^{\prime}\right)\right) \leq\left|x-x^{\prime}\right| / 2$ for every $x, x^{\prime} \in[0,2]$.

Example 4.3 We solve a linear (VRP) with the data $X, Y, A, P, Q$ and $q$ as given in the preceding example and with

$$
C=\left(\begin{array}{cc}
1 / 4 & -7 / 16 \\
0 & 0 \\
0 & 0
\end{array}\right), \quad c=\left(\begin{array}{c}
-11 / 16 \\
0 \\
2
\end{array}\right)
$$

Iteration 1 . We start the algorithm with $x_{0}=0$. The feasible set of the programs in Step 1 is given by the system $P y \leq q$ and consists of one element $\{0\}$. Hence the vector $b_{0}$ is equal to $(-11 / 16,0,2)^{T}$. In the next step, we minimize $|z|$ over the set $A z \leq b_{0}, z \in X$. The optimal solution is $z=11 / 16$. As $x_{0} \neq z$, it is not a solution of (VRP). 
Iteration 2 . We set $x_{1}=z=11 / 16$ and return to Step 1 to solve three programs whose feasible set is given by $P y \leq Q x_{1}+q$. We obtain $b_{1}=(-109 / 128,0,2)^{T}$. In Step 2 , we minimize $|11 / 16-z|$ over the set $A z \leq b_{1}, z \in X$ and obtain the optimal solution $z=109 / 128$. Since $x_{1} \neq z$, the point $x_{1}$ is not a solution of (VRP). If we choose $a$ priori a tolerance level $\epsilon=0.2$, then we may stop the algorithm and consider $x_{2}=109 / 128$ as an approximate solution of (VRP) because $\left|x_{1}-x_{2}\right|<\epsilon$. If not, we continue it with $x_{2}$ for restarting the procedure. It can be seen that the algorithm generates the sequence $\left\{x_{r}\right\}_{r=0}^{\infty}$ converging to $x=2$, which is the unique solution of (VRP).

\section{Competing interests}

The authors declare that they have no competing interests.

\section{Authors' contributions}

All authors contributed equally and significantly in writing this article. All authors read and approved the final manuscript.

\section{Author details}

${ }^{1}$ Faculty of Science, King Abdulaziz University, P.O. Box 80203, Jeddah, 21589, Saudi Arabia. ${ }^{2}$ Avignon University, Avignon, LMA-EA2151 84000, France.

\section{Acknowledgements}

This work was funded by the Deanship of Scientific Research (DSR), King Abdulaziz University, Jeddah, under grant No. 130-030-D1434. The authors, therefore, acknowledge with thanks DSR technical and financial support. The authors thank the referees for the valuable comments and appreciation.

Received: 29 July 2013 Accepted: 29 October 2013 Published: 25 Nov 2013

\section{References}

1. Luc, DT: An abstract problem in variational analysis. J. Optim. Theory Appl. 138, 65-76 (2008)

2. Agarwal, RP, Balaj, M, O’Regan, D: Variational relation problems in locally convex spaces. Dyn. Contin. Discrete Impuls. Syst., Ser. A Math. Anal. 18, 501-512 (2011)

3. Agarwal, RP, Balaj, M, O'Regan, D: A unifying approach to variational relation problems. J. Optim. Theory Appl. (2012). doi:10.1007/s10957-012-0090-x

4. Balaj, M: A fixed point-equilibrium theorem with applications. Bull. Belg. Math. Soc. Simon Stevin 17, $919-928$ (2010)

5. Balaj, M, Lin, LJ: Equivalent forms of a generalized KKM theorem and their applications. Nonlinear Anal. 73, 673-682 (2010)

6. Balaj, M, Lin, LJ: Generalized variational relation problems with applications. J. Optim. Theory Appl. 148, 1-13 (2011)

7. Balaj, M, Luc, DT: On mixed variational relation problems. Comput. Math. Appl. 60, 2712-2722 (2010)

8. Khanh, PQ, Luc, DT: Stability of solution sets in variational relation problems. Set-Valued Anal. 16, 1015-1035 (2008)

9. Lin, L, Ansari, QH: Systems of quasi-variational relations with applications. Nonlinear Anal. 72, 1210-1220 (2010)

10. Luc, DT, Sarabi, E, Soubeyran, A: Existence of solutions in variational relation problems without convexity. J. Math. Anal. Appl. 364, 544-555 (2010)

11. Pu, YJ, Yang, Z: Stability of solutions for variational relation problems with applications. Nonlinear Anal. 75, 1758-1767 (2012)

12. Pu, YJ, Yang, Z: Variational relation problems without the KKM property with applications. J. Math. Anal. Appl. 393 , 256-264 (2012)

13. Sach, PH, Tuan, LA, Minh, NB: Approximate duality for vector quasi-equilibrium problems and applications. Nonlinear Anal. 72, 3994-4004 (2010)

14. Dhara, A, Luc, DT: A solution method for linear variational relation problems. J. Glob. Optim. (2013, in press)

15. Granas, A, Dugundji, J: Fixed Point Theory. Springer, New York (2003)

16. Al-Homidan, S, Ansari, QH: Systems of quasi-equilibrium problems with lower and upper bounds. Appl. Math. Lett. 20, 323-328 (2007)

17. Mordukhovich, B: Variational Analysis and Generalized Differentiation. I. Basic Theory and II: Applications. Springer Berlin (2006)

18. Noor, MA, Al-Said, E: Iterative methods for solving general quasi-variational inequalities. Optim. Lett. 4, $513-530$ (2010)

19. Rockafellar, RT, Wets, RJ-B: Variational Analysis. Grundlehren der Mathematischen Wissenschaften, vol. 317. Springer, Berlin (1997)

20. Strodiot, JJ, Nguyen, TTV, Nguyen, VH: A new class of hybrid extragradient algorithms for solving quasi-equilibrium problems. J. Glob. Optim. (2011). doi:10.1007/s10898-011-9814-y

21. Mizoguchi, N, Takahashi, W: Fixed point theorems for multivalued mappings on complete metric spaces. J. Math. Anal. Appl. 141, 177-188 (1989)

22. Nadler, SB: Multi-valued contraction mappings. Pac. J. Math. 30, 475-488 (1969)

23. Aze, D, Corvellec, J-N: On the sensitivity analysis of Hoffman constants for systems of linear inequalities. SIAM J. Optim. 12, 913-927 (2002)

24. Bergthaller, C, Singer, I: The distance to a polyhedron. Linear Algebra Appl. 169, 111-129 (1992)

25. Bonnans, JF, Shapiro, A: Perturbation Analysis of Optimization Problems. Springer, New York (2000) 
26. Hoffman, AJ: On approximate solutions of systems of linear inequalities. J. Res. Natl. Bur. Stand. 49, $263-265$ (1952)

27. Li, W: Sharp Lipschitz constants for basic optimal solutions and basic feasible solutions of linear programs. SIAM J. Control Optim. 32, 140-153 (1994)

28. Zalinescu, C: Sharp estimation for Hoffman's constant for systems of linear inequalities and equalities. SIAM J. Optim. 14, 517-533 (2003)

29. Markin, JT: A fixed point theorem for set-valued mappings. Bull. Am. Math. Soc. 74, 475-488 (1968)

30. Ciric, L: Multi-valued nonlinear contraction mappings. Nonlinear Anal. 71, 2716-2723 (2009)

31. Feng, Y, Liu, S: Fixed point theorems for multi-valued contractive mappings and multi-valued Caristi type mappings. J. Math. Anal. Appl. 317, 103-112 (2006)

32. Klim, D, Wardowski, D: Fixed point theorems for set-valued contractions in complete metric spaces. J. Math. Anal. Appl. 334, 132-139 (2007)

33. Latif, A, Abdou, AAN: Multivalued generalized nonlinear contractive maps and fixed points. Nonlinear Anal. 74, 1436-1444 (2011)

34. Latif, A, Tweddle, I: Some results on coincidence points. Bull. Aust. Math. Soc. 59, 111-117 (1999)

35. Chang, TH: Fixed point theorems for multivalued mappings. Math. Jpn. 41, 311-320 (1995)

36. Daffer, PZ, Kaneko, H: Fixed points of generalized contractive multi-valued mapping. J. Math. Anal. Appl. 192, 655-666 (1995)

37. Jachymski, JR: On Reich's question concerning fixed points of multimaps. Boll. Unione Mat. Ital., A (7) 9, 453-460 (1995)

38. Suzuki, T: Mizoguchi-Takahashi's fixed point theorem is a real generalization of Nadlers. J. Math. Anal. Appl. 340 752-755 (2008)

39. Seeger, A: Eigenvalue analysis of equilibrium processes defined by linear complementarity conditions. Linear Algebra Appl. 292, 1-14 (1999)

10.1186/1687-1812-2013-315

Cite this article as: Latif and Luc: Variational relation problems: existence of solutions and fixed points of contraction mappings. Fixed Point Theory and Applications 2013, 2013:315

\section{Submit your manuscript to a SpringerOpen ${ }^{\odot}$ journal and benefit from:}

- Convenient online submission

- Rigorous peer review

- Immediate publication on acceptance

Open access: articles freely available online

- High visibility within the field

- Retaining the copyright to your article 